\section{THE PHARMACOLOGY AND THERAPEUTICS OF ICE.*}

By VICTOR G. L. FIELDEN. M.B., L.P.S.I., Demonstrator of Materia Medica and Pharmacy, Queen's College, Belfast, etc.

THe subject of this paper suggested itself to me as a result of hearing Dr. McKisack read notes of some cases of pneumonia which he had treated by the applications of ice; and the discussion which followed prompted some questions which have made me feel that a fuller consideration of the subject from a pharmacological point of view would be of benefit to the members of the Society.

\section{Pharmacorogy.}

Ice is used in the treatment of many inflammatory and other lesions, and the beneficial results obtained from it have marked it as a valuable therapeutic agent.

It may be employed:

(A) Internally, for example, by swallowing small pieces in the treatment of haematemesis, or sucking for the relief of thirst.

(B) Externally, either:

(a) Locally, as in the treatment of synovitis or of pneumonia ; or

(b) Generally, as a pack in treating hyperpyrexia, say, of typhoid or rheumatic fever.

Before, however, going into its therapeutics, I shall refer to its pharmacological effect.

(a) When used locally its action may be divided into:

(I) Superficial local or direct; and

(2) Deep local or reflex effect.

1. Superficial or direct local action is exemplified in the effect produced by the application of ice to inflamed piles or to prolapse of the rectum, also in its formerly more extensive use (either alone or with salt) as a local anaesthetic. Of course, a similar but milder effect may be obtained by an evaporating lotion, but with ice, or ice and salt, we can obtain the much greater effect becsuse of the much greater degree of cold. The four cardinal symptoms of inflammation are markedly diminished by a timely application of cold. (a) The pain is relieved because the smaller amount of blood to and effusion into the part causes less tension upon the nerve terminals ; $(b)$ the heat, and $(c)$ the redness, are lessened becanse of the contraction of the blood vessels; and $(d)$ the swelling is prevented or decreased on account of the lessened blood supply with consequent diminution of the effusion. The earlier the application the more pronounced will be the effect of the ice in cutting short or preventing the inflammatory process.

On the other hand, the inflammation may be of some duration, and the application of ice may not have the desired effect of relieving the pain, hest, and swelling, and, to some extent, of the redness. Why? The ice will so constrict the afferent and efferent vessels that the products of inflammation become imprisoned and the trouble may be increased, the first symptom complained of being discomfort, or increase of pain, instead of ease. This is, no doubt, the explanation of the empirical fact that the use of ice to an inflamed tissue only does good so long as it gives ease, whilst increase of pain or discomfort contraindicates its employment, and should be replaced by moist warmth, in order that the tissue may be flushed out and the inflammatory products carried off by a more liberal supply of b'ood. I must insist, however, upon an interval (longer or shorter, according to the extent of the trouble) between the removal of the ice and the application of heat, the interval being employed in gradually reducing the degree of cold, and then increasing the degree of heat (which is analogous to the treatment of frostbite), otherwise the remedy might possibly prove worse than the disease. A reversed graduation must be adopted if hot poultices be replaced by ice, else the sudden cold applied over dilated vessels, which have been further dilated by the heat, causes such a "water-logging" that moist gangrene be rapidly produced.

2. Deep Local or Reflex Effect. - When ice is applied to the chest wall, as, for instance, in the treatment of pneumonia, or to the abdominal wall for abdominal or pelvic inflammation, it is not for its direct effect one looks. I question if it would cause much, if any, direct constriction of even the superficial vessels of the subjacent viscus unless the wall was

* Read before the Ulster Medical Bociety. very thin. I am convinced that we are justified in considering the effect produced a reflex one upon the diseased organ, and not due entirely, if at all, to a general reduction of pyrexia as the result of the limited application of cold. In fact, it is analogous to, and probably identical with, that of counter-irritation. In a word, I think ice should be classed as a counter-irritant. What do we find writers on pharmacology and therapeutics say on the matter? Hale White writes: "The effects of a cold compress are the same as those of a linseed poultice." (A linseed poultice, I mav remind you is a rubefacient, or mild counter-irritant.) Hare says that the action of ice is "closely associated with the subject of counter-irritation." Sollman speaks of cold compresses and the icebag as "useful counter-irritants." An important fact in support of ice being counter-irritant is gleaned from a statement made by Ringer when referring to Arnott's use of ice and salt. He says: "If applied too long, this mixture may vesicate, but this will not occur under five or six minutes." Now the degree of counter-irritation obtained by a drug (whether it be rubefaction or vesication) is due to its potency, the duration of its application, and the greater or less delicacy of the skin to which it is applied. Therefore if ice and salt will cause vesication (an intense degree of counter-irritation), is ice not a milder counter-irritant? If this is so, let us briefly consider how a counter-irritant, for example, a linseed and mustard poultice, acts. Such an application does not produce a direct effect when applied over deep-seated disease such as pneumonia. The old idea that pneumonia, being a humoral disease, was benefited by a poultice drawing the "humour" from the lung to the surface, gave place to the theory that the hot poultice drew guch an amount of blood to the surface that it depleted the lung tissue. Were this latter correct, hot applications to any part of the body should be equally successful, but even the originator of the theory, I am sure, would hardly have treated pneumonia by poulticing the leg or other independent region.

A later theory was that the poultice not only dilated the cutaneous but, to an equal extent, the pulmonary capillaries by penetration of the heat. This could not be held good, for the application of a fly blister over the seat of disease produces an equally good, if not better, result than a poulticea result which could not be put down to moist warm th extending to the deeper tissues.

How, then, can the effect of hot applications to the surface in deep-seated inflammation be explained? By a reflex action through the vasomotor centres. This is believed to be the true explanation of the action of counter-irritants. The vasomotor centres are situated in the spinal cord and medulla, and when an impression is made upon the superficial vessels by counter-irritants (including ice) it is transmitted by the afferent nerves to the vasomotor centres and reflected to the viscera in association with that portion of the skin irritated for it has been pointed out "that a distinct relation exists between irritation of an internal organ and that part of the skin which is supplied by the same segment of the spinal cord or brain" (Head quoted by Cushney).

Foster states that there are few, if any, vaso-constrictor fibres to the skeletal muscles, although he mentions that "all or nearly all arteries of the body have vasomotor fibres." "The action of these vasomotor fibres is more manifest and probably more important in the case of small arteries than in large ones." However, vasomotor fibres are supplied to all the viscera. Those in the abdomen through the splanchnic nerves have long been known, but those of the thoracic contents are of recent acquaintance. Indeed, Foster says: "Concerning the vasomotor nerves of the thoracic viscera we know at present very little." And Schäfer, in his later work, says that proof of vasomotor control of the lung circulation is extremely difficult, so much so that some physiologists maintain that the vascular gystem of the lungs is not under the control of the nervous system. But, he continues, the evidence of Bradford and Dean, who have made most exhaustive research, appears conclusive in fayour of a vasomotor mechanism. Bradford and Dean found that vasomotor fibres for the lungs are from the third to seventh thoracic nerves inclusive. Schäfer also states that the pulmonary vascular system is undoubtedly innervated from the npper thoracic nerve roots, and thas comes under the influence of the central vasomotor mechanism. After quoting various experiments upon vaso. motor nerves and centres he says, "it is thus evident that the pulmonary circulation can be affected to a certain extent by sensory stimulation of the chest wall."

In order to get as pronounced an effect as possible upon the 
subjacent viscus, whether abdominal, thoracic, or cranial, a more or less extensive area of the skin is utilized for the action of the remedy, for "reflexes are more easily and more completely discharged when the specific end organ of the afferent (sensory) nerve is stimulated than when the trunk of the nerve is stimulated in its course" (Landois and Stirling). Chapman, however, as quoted by Ringer, has claimed excellent results by using an icebag over or along the spine. According to Foster the impulses transmitted to the vasomotor centres are not merely reflected, but "act afresh, as it were, as a stimulus to the nerve centre, producing, according to circumstances and conditions, either a few weak efferent impulses or a multitude of strong ones." Landois and Stirling make a similar statement in different words-" the vasomotor centre, therefore, primarily regulates the condition of the blood vessels, but through them it obtains its importance by regulating and controlling the blood supply, according to the needs of an organ."

I have endeavoured to show, by quoting from the works of recognized physiologists, that reflex action and the vasomotor mechanism are the important, if not the sole, factors in the effects produced upon deep-seated organs by counter-irritants applied to the skin.

Is it now possible to obtain an idea of what actually occurs in these deep-seated organs? There seems to be little doubt that when applied to the surface ice causes a reflex diminution of the blood supply to the viscus innervated from the same segme nt or segments of the spinal cord as that portion of skin to which the application is made. Bartholow points out that when one hand is immersed in cold water the temperature of the other falls by reflex action, and quotes that Brown-Séquard has experimentally shown that ice applied to the lumbar region causes contraction of the arterioles of the kidneys. Hale White says "cold appears to contract not only the vessels of the skin to which it is applied but also those of the organs under it. Most often this is reflex, but if the intervening tissues are thin it may be due to conduction, and perhaps this explains the utility of an icebag to the head for concussion and the fact that some writers -atrongly recommend ice poultices to the chest for pneumonia." And later on he repeats that cold contracts the vessels and owers the temperature at the point of application and in the parts beyond.

Exceedingly valuable observations were made by Rossbach apon the condition of the mucous membrane of the trachea by the application of ice to the abdomen of cats, and are recorded by him in his paper upon "the formation of mucus and the treatment of diseases of the mucous membranes in the air passages." In his studies of the effect of various remedies upon the mucous membrane of the air passages he devised the plan of actual inspection of the mucons membrane by laying open the whole of the trachea and keeping it exposed to view by retraction of the edges with hooks. Amongst the substances studied was included ice. After quoting his procedure and the effects noticed, he aays: "It may be concluded without doubt that the vessels of the mucous membrane of the larynx and windpipe can be infuenced in a marked degree in a reflex manner by strong irritation of cold applied to the skin. For we conclude from the quick appearance immediately after the application of the irritant that the primary contraction can only be a reflex result of the irritation of cold." The primary contraction he found was later followed by redness, and still later by a oluish-red colour, but contraction was again obtained, though not to so great a degree, by a renewed application of ice, which, he maintains, shows that the vessels are not paralysed. That vaso-dilator fibres are stimulated later than vasoconstrictor he does not consider the explanation, "for a stagnation (backwards) of the blood from the extreme degree of the second stage might rather point to that the arteries going to it might have remained contracted, and that the veins passing from it might be greatly dilated. or that only the capillaries were enormously dilated and the vessels to and from them had remained contracted."

It is evident, then, that when local contraction of the cutaneous vessels is caused by ice a reflex contraction of the arterioles occurs in the associated viscera. Had the reverse been the fact-namely, reflex dilatation caused by the local application of cold-it would have been impossible to explain the effect of an icebag to an inflamed tissue with but a thin covering, for example, in synovitis of the knee.joint in which both the direct and the reflex actions are concerned-direct contraction with reflex dilatation would indeed be a hard nut to crack. Having seen, however, that the reflex effect is con- traction of the arterioles, we are thoroughly able to understand that an icebag to an inflamed joint has both directly and reflexly just such an effect as I explained when dealing with the purely local action of ice-namely, reduction of the four cardinal symptoms of inflammation.

The same dual action (direct and reflex) comes into play without a doubt in the treatment of meningitis, etc., by an icebag to the head.

Besides these effects upon the local circulation it is necessary before passing to glance at other effects of ice. One of the chief and most beneficial is the rise of blood pressure which it canses, and so gives tone to the general circulation, including, of necessity, the heart. Respiration is stimulated, and, as pointed out by Ringer, cold causes the langs to absorb more oxygen. Again, has ice any beneficial effect upon inflammatory exudates? This is doubtless difficult to answer. Lees, writing in the Lancet, of 1889, stated that there was reason to believe that the icebag over pneumonic lung had a directly curative influence; that it did not simply reduce the general temperature, but that it distinctly tended to repress the inflammatory process in the lung with more or less success according to the severity of the case, and the height which the inflammation had already reached. Mays, in the same journal in 1893, spoke most highly of this same treatment, and stated that the resolving power of ice on the exudation is one of the strongest factors in its curative influence.

(b) General External Application.-When ice is employed for its general effect-for example, in the treatment of hyperpyrexia-its great action is that of abstraction of heat; but I cannot help believing that it has also a second or reflex action, according to the conditions present, upon the heat centres (the thermogenic thermolytic, and thermotaxic). My reason for this belief is that when the temperature is lowered by an icepack it is sometimes permanent, or, at any rate, the temperature may not rise so high as before the pack. Now, if it were simply a matter of abstraction of heat, the diminished temperature should be temporary, and on removal of the ice should gradually, and in a comparatively short time, rise again to its original height. Instead, however, it is found that a more or less permanent reduction is obtained which may last for days, and this could only be accounted for by a reflex effect produced upon the central nervous system. I admit that byperpyrexia may recur during an acute fever in spite of icepacks, but the explanation, doubtless, is that the illness is so acute that it affects to such a degree the heat centres, which had been benefited by the ice, that they once more give way under the strain and require further aid by another liberal use of ice. With an icepack the tonic effects to respiration and circulation already referred to are obtained to even a greater degree, and prove valuable in asthenic cases. This improved tone may be all that is necessary in many cases to ultimately bring to a successful termination an illness which might otherwise have proved fatal.

\section{Therapeutics.}

I have dealt at such length with the pharmacology of ice that I can only briefly refer to its therapeutics. We find it recommended in a great variety of conditions.

Internally. - Small lumps sucked prove most grateful to the patient parched with thirst, and also after operations upon the stomach. In the latter condition it is even more grateful than sips of water during the first twenty-four or forty-eight hours. It is recommended also in assisting to control haemorrhage from the mouth, throat, langs, and stomach. For haernatemesis, however, small pieces may be swallowed. In these conditions, not only is the action direct, but also markedly reflex. Iced drinks are more refreshing than the same liquids without the addition of ice. In painful conditions of the stomach pieces swallowed possess a sedative action, and they may prove valuable in vomiting from various causes.

Externally we may employ this valuable adjunct to treatment in the form of compress, poultice, icebag, or ice cradle. As a local anaesthetic prior to the opening of abscesses, etc., or for the performance of paracentesis it has fallen into disuse since the introduction of the ether spray and of ethyl and methyl chlorides. It is well to remember that a piece of ice dipped into salt and held against the skin will prove a valuable local anaesthetic should ethyl chloride, etc., not be available.

To the head it may be applied in the treatment of heat stroke, convulsions, delirium, headaches, otitis media, in certain cases of insomnia and in meningitis (simile and tuberculous). In meningitis the icecap is said to be "most 
efficacious," lowering temperature and modifying intracranial circulation. It is recommended to be kept applied till the temperature remains subnormal for some time. In the British Medical Journal of April 15th, 1899, Professor Lindsay reported the recovery of a case of probable tuberculous meningitis in which the main treatment was continuous application of cold to the head. Although continuous cold was obtained by using Leiter's tubes (and not an icebag), I refer to the case to illustrate the value of cold in the treatment of meningitis which may be almost doubtless tuberculous. The icecap is suggested in cerebral haemorrhage; but it is pointed out by Bartholow that it is harmful if the "face is pale, the surface cool. and the circulation depressed."

The icebag is useful in treatment of wounds of the eye to prevent inflammation, and is frequently employed in inflammatory diseases of that organ, and, as mentioned by Shaw, seems to do good so long as it allays the pain.

Dr. Gardiner Robb says that he has found ice to the face in small-pox give greater relief than any other application. In spinal and cerebro-spinal meningitis ice may be applied along the whole length of the spine, and has been found of great service. In this case there is (as when applied to the head) both a direct and a reflex action.

Ringer recommends ice poultices to the throat in tonsillitis, scarlet fever, and diphtheria, especially if the glands are likely to suppurate.

To the chest in the cardiac region ice is of great benefit in treating pericarditis, and some writers speak of its usefulness in endocarditis. It has been found to give great relief in the palpitation of exophthalmic goitre, and of functional heart troubles.

More extensive application to the chest has given decidedly beneficial results in cases of haemoptysis. I have already spoken at some length of the use of ice in pneumonia. Several writers have seen no bad symptoms from its use, and the existence of pneumonia is no contraindication to the use of icepacks for hyperpyrexia. As it was a paper on pnea. monla treated by ice applications which led me to write this paper, I should like to briefly quote some statistics regarding mortality in this disease. I have not found any more recent than those quoted by Mays in the Lancet of July 8th, 1893. He collected 50 cases treated with ice applications of which 2 were fatal - that is, a mortality of 4 per cent. Besides these, he quotes from a paper by Fieandt, a Finnish medical man, who treated 106 cases (10 of which were double pneumonia), with 3 deaths, equal to 2.82 per cent. Together these constitute 156 cases with 5 deaths-that is, a mortality of 32 per cent. In comparisou with these figures Osler puts the mortality at from 20 to 40 per cent. In the Johns Hopkins Hospital it was 29.8 per cent. Taylor estimates the mortality at 17 per cent. Certainly a striking difference in favour of ice.

I have not found any statistics regarjing the efficiency of early as compared with late application of ice in this disease, but theoretically I should imagine that a more beneficial action would be obtained if applied in the early stage, when we might expect a smaller amount of inflammatury exudation to be thrown out. On the other hand, if consolidation has already occurred, it might be found that moist warmth by flushing the blood vessels would tend to a more rapid absorption of the effused products. I am unable to speak from personal experience, but make this suggestion from theoretical considerations.

The treatment of pneumonia by the ice cradle and not by direct application to the skin has been used, and in 43 cases treated by this method the mortality was 7 per cent. For its mode of use I refer my hearers to Sir William Whitla's Dictionary of Treatment. In the Medical Annual of 1899 it is pointed out that if ice is used in the treatment of pneumonia the patient needs careful watching; if the feet are cold hot bottles must be used; if the temperature falls below $99^{\circ}$, and the hands are cold or the lips bluish, the icebag should be removed.

The pains associated with pleuritic complications or affections are frequently greatly relieved with ice, but in some cases have been found to be aggravated.

To the abdominal wall ice has been employed in treating appendicitis and other painful inflammatory conditions of the viscera and peritoneum ; also in splenic hypertrophy, haematemesis, and for the relief of vomiting from various causes. It has also been found of great service in haemorrhage from the bowel or uterus, and in the latter case pieces may be introduced into the uterns or rectum.

Locally, ice may greatly benefit painful conditions of the testis, and is recommended to assist reduction of prolapge of the uterus and rectum. It has been found useful in some cases of lumbago, also to assist in the reduction of strangulated hernia, and for the relief of haemorrhoids which are swollen, painful, and bleed.

To the limbs the icebag may be applied when neuralgia of various nerves requires immediate relief by active treatment, but it is for inflammations in joints and bursae that we find the icebag more frequently pressed into service. After excisions of joints and other operations upon bone the application of an icebag over the dressings will greatly diminish the oozing which is sure to occur, as well as lessen the pain which might otherwise follow. As an alternative to moist warm th ice may be emplnyed in the expectant treatment of osteitis, myositis, and inflammation of other tissues prior to the formation of pus.

An icepack to the trunk or trunk and limbs will be found of the utmost value, as already mention ed, in treating hyperpyrexia occurring during typhoid, acute rheumatism, scarlatina, and other febrile conditions. Even if pneumonia occurs as a complication the icepack is not contraindicated. The rectal temperature must be watched, and when it falls to $100^{\circ}$ the pack must be removed, when a still further decrease will be noticed. If kept on too long collapse is liable to occur.

\section{THE SANATORIUM TREATMENT OF PHTHISIS :} IS IT WORTH WHILE?

AN ANSWER FROM THE WESTMORLAND SANATORIUM.

By WILLIAM S. PAGET-TOMLINSON, M.D.LoND., M.R.C.P.,

Treasurer of the Sanatorium.

IT seems very desirable that all who have had any considerable experience in this matter should, at the present time, record the conclusions at which they have arrived. My chief interest in, and practical experience of the question having been almost entirely in connexion with the consumptive poor at our Westmorland Sanatorium, it will be readily geen that I have no pecuniary axe to grind, but simply desire to arrive at the truth concerning the value of the treatment-a subject which is just now agitating the professional mind. My knowledge of sanatoriums for the well-to-do classes is limited to visits paid to various institutions in this country and to a study of their statistics, but I have followed much more closely the work of such sanatoriums for the poor as exist in Great Britain, and especially, of course, that of our own in Westmorland, which was one of the first in England to deal with the poor upon any extensive scale. The result of its five years' work is briefly recorded in the British MEdical Journal of March 4th, but of this more presently.

To me it seems incredible that any one having any considerable acquaintance with the subject should speak of the treatment as being productive of no good, and indeed positively harmful. It is doubtless true that seven or eight years ago an exaggerated estimate was probably formed as to the extraordinary cures that would be made. This is not surprising; it is nearly always so with any new method of treatment. A few years' experience, however, has taught us our limitations, and although we may have had a few disappointments in connexion with the treatment, I unhesitatingly affirm my belief that not only does the sanatorium treatment hold the field, but I would go further and say that I strongly suspect that other methods of cure, brought forward from time to time, owe their chief success to observance of openair principles as an adjunct to whatever other means may be adopted.

I entirely agree with the views expressed by Dr. Ransom, of Nottingham, in a paper published in the BRITISH MEDICAL JOURNAL of January 14th, 1905, that we were prohably, in the first instance, a little too sanguine, and chiefly in three directions: (1) As to the general applicability of the treatment; (2) as to the time required to arrest the disease; (3) as to the permanence of the arrest.

With regard to the first of these, quite apart from the particular stage of the disease. it would geem that there are some cases which do not respond to treatment as we should expect, owing to causes not always easy to determine; as to the second point, it is certain that cases (even early ones) require a longer period of treatment than was originally expected; if this be kept in view, there will be a far better chance of fulfilment of the third condition-permanence of arrest-though in 